\title{
Demographics, Personality and Substance-Use Characteristics Associated with Forming Romantic Relationships
}

\author{
Eilin K Erevik $^{1}$ - Ståle Pallesen ${ }^{1} \cdot$ Cecilie S Andreassen ${ }^{2,3} \cdot \varnothing_{\text {ystein Vedaa }}^{4,5} \cdot$ Anders Skogstad $^{1} \cdot$ Amandeep Dhir $^{6}$. \\ Torbjørn Torsheim ${ }^{1}$
}

Published online: 11 July 2019

(C) The Author(s) 2019

\begin{abstract}
The current study aimed to identify demographic, personality and substance-use characteristics associated with forming romantic relationships. Data were collected by two online surveys among students in Bergen, Norway, during the autumn of 2015 (T1) and by a follow-up survey that was conducted 12 months later (T2). The current sample consists of the 2404 participants who reported being single at T1 (mean age 23.2) and who participated in both waves of the survey. Binary logistic regression analyses were conducted. Separate analyses were conducted for both sexes and for the entire sample of participants. High extroversion scores predicted relationship formation. For women, having a child/children, higher scores on neuroticism, alcohol use and illegal substance use positively predicted relationship formation, while for men, age and openness were positive predictors. The study contributes with several novel findings. In general, characteristics related to a need for support predicted romantic relationship formation among women, while characteristics associated with increased resource acquisition potential predicted relationship formation among men. The general pattern of findings is in line with established evolutionary theories such as the sexual strategies theory and the parental investment theory.
\end{abstract}

Keywords Romantic relationships $\cdot$ Students $\cdot$ Personality $\cdot$ Substance use $\cdot$ Parental status $\cdot$ Sex

Romantic relationships are typically considered as relatively long-term, mainly monogamous commitments between two individuals. Romantic relationships play a pivotal role in human societies and seem to be preferred over short-term mating (Lovejoy 1981; Waal 2006). Individuals in romantic

Eilin K Erevik

eilin.erevik@uib.no

1 Department of Psychosocial Science, University of Bergen, PO. Box. 7807, 5020 Bergen, Norway

2 Department of Clinical Psychology, University of Bergen, PO. Box. 7807, 5020 Bergen, Norway

3 Department of Social Studies, University of Stavanger, PO. Box. 8600 Forus, 4036 Stavanger, Norway

4 Department of Health Promotion, Norwegian Institute of Public Health, PO. Box. 973 Sentrum, 5808 Bergen, Norway

5 Department of Mental Health, Norwegian University of Science and Technology, PO. Box. 8905, 7491 Trondheim, Norway

6 Department of Teacher Education, University of Helsinki, PO. Box. 9, Siltavuorenpenger 5 A, 00014 Helsinki, Finland relationships tend to be healthier and live longer than single individuals suggesting that pair-bonding may involve survival advantages (Averett et al. 2008; Kiecolt-Glaser and Newton 2001). Several evolutionary explanations have been suggested as to why humans commonly arrange intersex mating through monogamous romantic relationships. For one, monogamy may have reduced infanticide, as being close to the mother and offspring would enable the biological father to protect the offspring from infanticidal males (Opie et al. 2013). Monogamy may also have increased the offspring's likelihood of survival in general, as having two caretakers would imply more resources and protection compared to having only one caretaker (Opie et al. 2013). Furthermore, monogamy may have been adaptive through reducing same-sex competition, hence fostering same-sex cooperation and increasing the likelihood of group survival (Desmond 1967; Waal and Gavrilets 2013). Finally, some evolutionary scholars have argued that monogamy may have evolved because food shortage forced women to live quite isolated from their group of origin (Lukas and Clutton-Brock 2013; Waal and Gavrilets 2013). This isolation of women would make a long-term mating strategy adaptive for men, as short-term and/or polygamic mating 
strategies would involve too much migration (Lukas and Clutton-Brock 2013). Evolutionary research on romantic relationships has traditionally centred on opposite-sex couples, but same-sex romantic relationships are suggested to entail survival and reproductive advantages as well (Kirkpatrick et al. 2000).

There are individual differences in the ability/tendency to engage in romantic relationships. Moreover, an increasing percentage of single and childless individuals in many Western and Asian societies have raised concern about the sustainability of social welfare systems (Adamczyk 2017; Nargund 2009). Knowledge of characteristics predicting relationship formation may be conducive if one wishes to understand the mechanisms promoting relationship formation and pregnancies. From an evolutionary perspective, one can expect factors such as demographics, personality and substance use to predict who forms romantic relationships (Buss 2007, 2009; Petraitis et al. 2014). Individual characteristics may affect the likelihood of forming a romantic relationship in three main ways. Firstly, individual characteristics relate to mate value, where potential mates perceive some characteristics (e.g. physical attractiveness) as compelling traits (Buss 2007). Secondly, individual characteristics may affect the individual's motivation for different mating strategies (i.e. shortterm versus long-term mating strategies) (Buss 2007). For instance, paternal absence during childhood has been found to predict short-term mating strategies (Draper and Harpending 1982). Finally, some individual characteristics, like humour, may make the individual better equipped to chase off same-sex competitors and consequently make the person more successful at securing a long-term mate (Buss 1989). Existing research has primarily investigated the mate value of different individual characteristics, while the associations between individual characteristics and actual relationship outcomes have received less attention.

\section{Demographics}

Sex is the most studied individual characteristic associated with relationship outcomes, where sex is theorised and found to affect both choice of mating strategy and attractiveness judgements (Buss 1989). According to the well-known and acknowledged sexual strategies theory, which is supported by several empirical studies, men will prefer a short-term sexual strategy more often than women. Further, relatively to each other, men will emphasise signs of fertility and reproductive value (i.e. expected future reproduction) in a potential mate while women will emphasise a mate's ability and willingness to provide resources (Buss 1989; Buss and Schmitt 1993). Sex differences in mating strategies and attractiveness judgements are commonly attributed to women's increased parental investment and limited reproductive capacity and to men's uncertainty regarding paternity and their extensive reproductive potential (Buss 1989; Trivers 1972). In addition to sex, a range of other demographic characteristics may also predict romantic relationship formation through influencing mate value, mating strategy and/or capacity for same-sex competition.

Men are theorised to value fertility and reproductive value highly, and women as well, to some extent, when seeking long-term mates (Buss and Schmitt 1993; Soler et al. 2003). Both fertility and reproductive value (i.e. the number of future offspring an individual can produce) decrease with age, in particular among women (Frank et al. 1994; Kidd et al. 2001). For women, fertility will increase from menarche, peak in early to mid-20s and decrease after this (slowly at first) until it dissolves around menopause (i.e. average age of onset is 50 51 years), while reproductive value will peak in mid-teens and dissolve with menopause (Buss and Schmitt 1993; Frank et al. 1994; Wallace and Kelsey 2010). For men, fertility in relation to age is less studied, but it is suggested that men's fertility will peak in their early to mid-30s (Levitas et al. 2007). Further, the decline in fertility is less steep for men compared to women, and men's capacity for reproduction have no definite endpoint (Buss and Schmitt 1993; Kidd et al. 2001). Men's reproductive value will in general be rather high throughout their lifespan despite the decrease in fertility. Men's high reproductive value throughout the lifespan is explained by the low minimum investment necessary for a man's reproduction which involves that men at most ages in theory could produce a very high number of offspring if they were to mate with numerous, fertile women (Buss 1989; Trivers 1972). However, both men's and women's capacity to provide for their offspring is likely to be reduced in older age due to health issues and reduced earning capacity. The age-dependent decrease in fertility and reproductive value (and/or capacity to provide for offspring) suggest that older adults may have a lower mate value and perhaps a lower likelihood of entering romantic relationships compared to younger adults. Accordingly, a study found increasing age to reduce the likelihood of forming romantic relationships (Skew et al. 2009). In addition to indicating decreased fertility, increasing age, up until a certain point, may also indicate more resources (e.g. higher salaries) (Buss 1989). Women have been found to especially value characteristics that may indicate that a mate has more available resources (Buss 1989). Due to women's steeper decrease in fertility and reproductive value with advancing age (which may decrease their mate value), along with the fact that women seem to place greater emphasis on a mate's resources (which may make women more accepting of older men), women's likelihood of finding a long-term mate is hypothesised to decrease more with age compared to men's (Buss 1989). In addition to evolved preferences for long-term partners of a certain age, cultural factors like norms and the arrangement of maternity leave are also factors likely to affect when (or if) 
people seek committed relationships (Dixon 1971; Manning et al. 2014).

Country of birth is another demographical characteristic that may predict relationship status. Generally individuals similar to each other are more likely to become romantically involved, a tendency which also holds true in terms of cultural background (Botwin et al. 1997; Qian et al. 2012). Accordingly, one might expect members of the majority culture/nationality to have better chances of finding a partner within the majority culture compared to members of minority cultures. From an evolutionary perspective, assortative mating is often explained as humans seeking mates with similar phenotypic traits - a tendency which has been suggested to be adaptive because similar phenotypic qualities increase the likelihood of shared genotypic characteristics. Shared genotypic characteristics will further result in greater genetic relatedness between parent and offspring and hence increased genetic survival (Hamilton 1964; Thiessen and Gregg 1980). Increased genetic relatedness within the family is further theorised to increase altruism and hence the social bond within the family, which is in line with the inclusive fitness theory and findings that suggest genetic relatedness to foster altruism (Hamilton 1964; Thiessen and Gregg 1980). The tendency to seek similarity, however, is assumed to have an upper limit as to avoid inbreeding (Thiessen and Gregg 1980).

Finally, the likelihood of forming a romantic relationship may also be predicted by parental status. Having children from another relationship may signal reproductive capacity and willingness to invest in offspring, but may also suggest that the mother/father has less available resources for a new partner and additional children (Sommer et al. 2013). Studies have found that single individuals who have children are rated as less attractive by potential mates and have a lower probability of getting married or finding a new partner (Bernhardt and Goldscheider 2002; Plumm et al. 2016; Skew et al. 2009; Sommer et al. 2013). Single women who have children are further rated as less attractive compared to single men who have children (Sommer et al. 2013).

\section{Personality Factors}

Personality factors reflect stable tendencies in the way an individual feels, thinks and acts and are considered determinants of a range of life outcomes, including relationship outcomes (Roberts et al. 2007). The five-factor model, encompassing extroversion, agreeableness, conscientiousness, neuroticism and openness, is the most acknowledged and applied taxonomy of personality traits (Larsen et al. 2013; McCrae and John 1992). Individual differences in the five-factor model's personality traits, as well as a sensitivity for detecting such differences, are thought to have evolutionary underpinnings (Buss 2009; Nettle 2006). Personality traits are likely to affect mate value because they indicate how one would function as a partner and a parent (Botwin et al. 1997; Nettle 2006). High scores on extroversion, agreeableness, conscientiousness and openness and low scores on neuroticism are generally perceived as attractive (Botwin et al. 1997; Figueredo et al. 2006). Still, a limited number of studies have investigated whether personality traits predict romantic relationship formation, and those studies yield inconsistent results. One longitudinal study found no significant associations between relationship status and the five-factor model's personality traits (Shaver and Brennan 1992). Another study found higher scores on sociability (i.e. a sub-facet of extroversion) and neuroticism to predict relationship formation among young adults (Neyer and Lehnart 2007), whereas another study, in which personality was assessed post hoc, found men with high scores on neuroticism to be less likely to be in a romantic relationship at the age of 40 (Skirbekk and Blekesaune 2014).

\section{Substance Use}

Humans have consumed psychoactive substances (in the form of fruits and plants) since ancient times (Dudley 2002; Sullivan and Hagen 2002). Consuming fruits and plants with psychoactive properties may have been adaptive due to these fruits/plants' nutritional components and because they may have helped consumers deal with extreme stress and fatigue (Dudley 2002; Sullivan and Hagen 2002). Given our long history of substance use, one may hypothesise that this behaviour is related to sexual selection as well (Dudley 2002). It is conceivable to assume that substance use could relate to both mate value, preferred mating strategy and the number of opposite sex competitors in ones' milieu. Substance use may increase mate value through signalling a willingness to take risks (Petraitis et al. 2014). A willingness to take risks may be perceived as attractive, specifically in men, as it may signal a better capacity to provide for a partner and offspring (Bassett and Moss 2004; Farthing 2005; Henderson et al. 2005). Further, in accordance with the handicap principle and the costly signalling theory, substance use might be interpreted as a signal of good genes as only individuals with particularly good genes may afford to take such risks (Farthing 2005; Zahavi 1975). One may, however, expect excessive substance use to be perceived as unattractive, as excessive substance use is associated with poor health - a trait that both sexes perceive as unattractive (Anthony et al. 1989; Grant et al. 2004; LevRan et al. 2014; Rehm et al. 2003; Rehm et al. 2006; Shackelford et al. 2005). Substance use could also be reasoned to be associated with either an increased or a decreased motivation to find a long-term partner. On the one hand, heavy substance use may involve a greater need for emotional support and hence a larger motivation to find a long-term partner (Anthony et al. 1989; Grant et al. 2004; Lev-Ran et al. 2014). 
On the other hand, those seeking short-term mates may use substances as means to gain access to or to muster the courage to approach potential short-time mates as one-night stands typically occur in substance use settings (Pedersen et al. 2017; Taylor et al. 1999). Finally, substance use may affect same-sex competition. Men tend to use substances to a greater extent than women (Erevik et al. 2017a, 2017b), and women may hence have fewer same-sex competitors in settings where substances are used, whereas men may have more competitors in such settings.

Few studies have investigated substance use as a predictor of relationship outcome, and the findings from these studies are equivocal. Most studies have found substance use to be a negative predictor of relationship formation, but some studies have found substance use to be a positive predictor of relationship formation and still others have found no association between substance use and later relationship outcomes (Bachman et al. 1997; Fu and Goldman 1996; Kandel et al. 1986; Leonard and Rothbard 1999; Newcomb and Bentler 1987). Limitations of these previous studies include that the relationship outcome has mainly been marriage and the study participants have been quite young at the time of the outcome measurement (i.e. typically early twenties). It is reasonable to expect that individuals who marry in their early twenties have certain characteristics (e.g. religiosity) and these characteristics may explain the observed inverse associations between substance use and marriage. There is a lack of studies investigating substance use as a predictor of relationship formation in general (i.e. not constrained to marriage) in older populations.

\section{Study Objectives}

Overall, few studies have investigated the relationship between demographics, personality traits and substance-use characteristics and actual relationship outcomes. Furthermore, previous studies have not included demographics, personality traits and substance-use characteristics in the same model, which is a limitation as these characteristics covary (Erevik et al. 2017a, 2017b; McCrae 2002; McCrae et al. 1999). Including demographics, personality traits and substance-use characteristics in the same model can thus increase the certainty that identified predictors explain unique proportions of the variance in relationship formation, rather than just being covariates of predictors not included in the model. Against this backdrop, we aimed to investigate demographics (i.e. age, country of birth and parental status), personality traits (i.e. the five-factor model's personality traits) and substance-use characteristics (i.e. nicotine, alcohol and illegal substance use) as potential predictors of change in relationship status, from single to a romantic relationship within 1 year. Separate analyses and interaction tests based on sex were run to account for the well-established sex differences in preferences and mating strategy.

\section{Methods}

\section{Procedures and Sample}

Data were collected by two online surveys. The first survey was sent by e-mail to all students at the four largest institutions for higher education in Bergen, Norway, during fall 2015 (T1). A total of 11,236 (39.4\%) students participated at T1. The second survey was sent out by e-mail during fall 2016 (T2) to those who participated at T1. A total of $5217(51.5 \%)$ participated at $\mathrm{T} 2$. In the current study, the sample consists of the 2404 participants who reported to be single at T1 and who participated in both surveys. The $\mathrm{T} 1$ response rate for the current sample is not known because we do not know the proportion of single students in the student population. The $\mathrm{T} 2$ response rate for those who reported being single at $\mathrm{T} 1$ was $45.8 \%$. In the sample, $20.2 \%$ (20.6\% of the women and $19.5 \%$ of the men) reported to be in a romantic relationship at T2. Sample characteristics are shown in Table 1. Informed consent was obtained from all participants included in the study. The study was approved by the Regional Committee for Medical and Health Research Ethics, health region Western Norway (project number 2015/1154).

\section{Measurements}

Demographics were measured at T1, including sex, age, country of birth and parental status. Participants were asked to specify their relationship status (i.e. single; steady romantic partner, but living alone; in a cohabitant relationship; married/registered partnership; other) at both T1 and T2 (Nedregård and Olsen 2014).

Personality was assessed at T1 with the well-validated short form of the International Personality Item Pool (MiniIPIP; Donnellan et al. 2006). Mini-IPIP includes 20 items, of which four items reflect each of the five-factor model's personality traits. Total scores for each trait range from 4 to 20 . In the present study, the Cronbach's alphas of the items measuring extroversion, agreeableness, conscientiousness, neuroticism and openness were $0.83,0.77,0.69,0.75$ and 0.74 , respectively.

The following types of substance use were measured at $\mathrm{T} 1$. Nicotine use was measured by single items assessing the frequency of tobacco smoking and the frequency of which the respondents used "snus" or other nicotine products. "Snus" is a nicotine product, resembling dipping tobacco which is primarily used in Norway, Sweden and Iceland. Alcohol use was assessed by the Alcohol Use Disorders Identification Test (AUDIT; Babor et al. 2001; Bohn et al. 1995). The AUDIT 
Table 1 Sample characteristics, $N=2404$

\begin{tabular}{|c|c|c|c|c|c|}
\hline \multirow[b]{2}{*}{ Characteristics T1 } & \multirow{2}{*}{$\begin{array}{l}\text { Full sample } \\
\text { Mean }(S D) / \%\end{array}$} & \multicolumn{2}{|l|}{ Women } & \multicolumn{2}{|l|}{ Men } \\
\hline & & $\begin{array}{l}\text { Single T1 and T2, } \\
n=1183 \\
\text { Mean }(S D) / \%\end{array}$ & $\begin{array}{l}\text { Single } \mathrm{T} 1 \text {, romantic } \\
\text { relationship } \mathrm{T} 2, n=307 \\
\text { Mean }(S D) / \%\end{array}$ & $\begin{array}{l}\text { Single T1 and T2, } \\
n=736 \\
\text { Mean }(S D) / \%\end{array}$ & $\begin{array}{l}\text { Single T1, romantic } \\
\text { relationship T2, } n=178 \\
\text { Mean }(S D) / \%\end{array}$ \\
\hline \multicolumn{6}{|l|}{ Demographics } \\
\hline Age & $23.2(4.5)$ & $23.1(4.7)$ & $23.0(4.3)$ & $23.4(4.1)$ & $24.0(4.9)$ \\
\hline Women & 62.0 & - & - & - & - \\
\hline Born in Norway & 93.8 & 94.3 & 95.1 & 92.7 & 93.8 \\
\hline Have child/children & 2.6 & 2.9 & 4.6 & 1.5 & 2.3 \\
\hline \multicolumn{6}{|l|}{ Personality ${ }^{\mathrm{a}}$} \\
\hline Extroversion & $13.9(3.8)$ & $14.1(3.7)$ & $14.8(3.4)$ & $13.1(3.9)$ & $14.9(3.6)$ \\
\hline Agreeableness & $16.8(2.8)$ & $17.4(2.4)$ & $17.6(2.2)$ & $15.5(3.2)$ & $16.1(2.8)$ \\
\hline Conscientiousness & $14.4(3.2)$ & $14.6(3.2)$ & $14.4(3.1)$ & $14.0(3.3)$ & $14.2(3.1)$ \\
\hline Neuroticism & $10.9(3.6)$ & $11.5(3.4)$ & $12.0(3.7)$ & $9.7(3.4)$ & $9.3(3.8)$ \\
\hline Openness & $14.6(3.2)$ & $14.1(3.2)$ & $14.4(3.1)$ & $14.9(3.2)$ & $16.0(2.8)$ \\
\hline \multicolumn{6}{|l|}{ Substance use } \\
\hline Daily nicotine use & 20.6 & 17.3 & 19.9 & 24.3 & 28.1 \\
\hline No alcohol use & 6.2 & 7.1 & 2.4 & 7.3 & 2.4 \\
\hline Low-risk alcohol use $^{\mathrm{b}}$ & 31.9 & 37.5 & 32.5 & 26.3 & 15.9 \\
\hline Hazardous alcohol use ${ }^{c}$ & 52.5 & 49.2 & 55.9 & 52.1 & 65.9 \\
\hline Harmful/dependent alcohol use ${ }^{\mathrm{d}}$ & 9.7 & 6.2 & 9.2 & 14.2 & 15.9 \\
\hline Illegal substance use last 6 months & 16.8 & 10.1 & 16.8 & 24.0 & 31.5 \\
\hline
\end{tabular}

$T 1$ time of the first survey, $T 2$ time of the second survey, $S D$ standard deviation. ${ }^{\text {a }}$ Total scores range from 4 to 20 for each trait, ${ }^{\mathrm{b}} 0<\mathrm{AUDIT}<8,{ }^{\mathrm{c}} 7<$ AUDIT $<16,{ }^{\mathrm{d}}$ AUDIT $>15$

includes 10 items assessing alcohol consumption, alcoholrelated harm and alcohol dependency symptoms. Total scores range from 0 to 40 . Respondents obtain a score of 0 if they report no consumption of alcohol during the preceding year, and no previous experiences with being injured, causing injuries to others, or causing others to be anxious as a result of their own alcohol use. Scores between 1 and 7 indicate lowrisk alcohol use; scores of 8 or higher suggest hazardous alcohol use. Scores of 16 or higher indicate harmful alcohol use, and scores of 20 or higher indicate dependent alcohol use (Babor et al. 2001). In the present study, the AUDIT obtained a Cronbach's alpha of 0.78. Illegal substance use was assessed by asking the respondents if they had ever used illegal substances or prescription drugs without a personal prescription. Those who confirmed lifetime use were further asked how many times during the last 6 months (i.e. never; used previously, but not the last 6 months; 1-4 times; 5-50 times; more than 50 times) they had used a range of different drugs (i.e. cannabis; synthetic cannabis; amphetamine/ methamphetamine; ADHD medication (without prescription); cocaine (crack); ecstasy; LSD/hallucinogens; heroin; synthetic heroin (without a prescription); sedatives (without a prescription); inhalants (e.g. paint thinner, glue); anabolic steroids; other illegal drugs or prescription drugs without a prescription) (Nedregård and Olsen 2014).

\section{Analyses}

Three adjusted (for all independent variables) binary logistic regression analyses were conducted: one including only the female participants, one including only male participants and one including the full sample. In the full sample analysis, we tested for interaction effects between sex and the other independent variables. The dependent variable in the regression analyses was change in relationship status from single at $\mathrm{T} 1$ to a romantic relationship at $\mathrm{T} 2$, while being single at both $\mathrm{T} 1$ and $\mathrm{T} 2$ was the reference category. The independent variables were age, country of birth (Norway vs. other countries), parental status (have child/children vs. no child/children), extroversion, agreeableness, conscientiousness, neuroticism, openness, nicotine use (daily vs. non or non-daily nicotine use), alcohol use (low-risk, hazardous and harmful/dependent alcohol use vs. no alcohol use) and illegal-substance use (illegalsubstance use during the previous 6 months vs. no illegalsubstance use last 6 months). Only T1 variables were used as independent variables because we wanted to identify temporal predictors of relationship formation. Thus, the inclusion of T2 variables as independent variables could have hampered the establishment of directionality as relationship formation is known to alter some of the included variables (e.g. personality traits) (Neyer and Asendorpf 2001; Neyer and Lehnart 2007). 
Harmful and dependent alcohol use were collapsed into one category because relatively few students reported such alcohol use (i.e. $7.0 \%$ and $2.7 \%$, respectively). The continuous independent variables were transformed into $z$-scores to enable comparison of the impact of the different independent variables against each other. Conservative requirements such as having a sample of at least 500 cases and at least 10 cases per predictors were met (Long 1997). Missing data were deleted listwise. In the full sample analysis, 258 (10.7\%) cases were excluded because they did not complete the survey at $\mathrm{T} 1$ and thus had missing data on some of the included variables, whereas $100(10.9 \%)$ cases were excluded from the analysis including only the male participants, and $158(10.6 \%)$ cases were excluded from the analysis including only the female participants. Listwise deletion was chosen because the missing data were exclusively on the predictor variables and in such instances listwise deletion in logistic regression may cause less biased results compared to other common approaches for handling missing data (Allison 2001, 2014). The associations between the independent variables and the dependent variable were reported as odds ratios (ORs). OR is considered as an effect size, but interpreting the magnitude of ORs is exacting, as the interpretation depends on the rate of the dependent variable (Chen et al. 2010) as well as the number of levels of the predictor. ORs of 2.0, 3.0 and 4.0 have been suggested to indicate small, moderate and large effect sizes, respectively (Ferguson 2009), but Ferguson (2009) emphasises that ORs below 2.0 are hard to interpret in terms of effect size.

\section{Results}

The results of the binary logistic regression analyses are shown in Table 2. All the associations presented below were statistically significant at $p<0.05$.

Age was positively associated with being in a romantic relationship at $\mathrm{T} 2$ among male students, but not among female students. The sex-by-age interaction effect was significant. Female students who had children were more likely to have entered into a romantic relationship at $\mathrm{T} 2$ compared to female students without children.

Extroversion was positively associated with having formed a romantic relationship at $\mathrm{T} 2$ for both female and male students. Neuroticism was positively associated with entering a romantic relationship among women, but not among men. Sex had a significant interaction effect on the relationship between neuroticism and forming a romantic relationship at $\mathrm{T} 2$. Openness was positively associated with being in a romantic relationship at $\mathrm{T} 2$ in the male group, but not in the female group. The sex-by-openness interaction was significant.

Among women, low-risk, hazardous and harmful/ dependent drinkers were more likely to have entered a romantic relationship at $\mathrm{T} 2$, compared to female non-drinkers. Among women, those reporting illegal substance use during the last 6 months at $\mathrm{T} 1$ were more likely to be in a relationship at T2, compared to the women who did not report illegal substance use during the previous 6 months.

Most of the effect sizes were small or very small (i.e. <3.0), although it should be mentioned that ORs below 2.0 are hard to interpret in terms of magnitude (Ferguson 2009). The associations between having children and harmful/dependent alcohol use and forming romantic relationships among women had moderate effect sizes.

\section{Discussion}

The findings from the current study can help delineate those who stay single, perhaps involuntarily. Being single may in some instances have detrimental effects on the individual's quality of life (Dush and Amato 2005; Soons and Liefbroer 2008). Knowledge about characteristics associated with remaining single can thus be of great societal importance, because knowing why some people stay single might be pivotal if one wishes to develop strategies to reduce the proportion of single individuals. The current study identified several individual characteristics that predicted the transition from being single to being in a relationship over a 1 -year period. Most of the significant associations had small effect sizes but given the immense importance of relationship outcomes and the difficulties associated with interpreting small ORs, these effects may still be of theoretical and/or practical importance. Many of the findings in the current study have not been reported elsewhere. In the following discussion, we will first consider possible explanations for why each of the identified characteristics was related to romantic relationship formation in general, before speculating on possible reasons for the sex differences.

\section{Age and Parental Status}

The finding that increasing age predicted romantic relationship formation among men stands in contrast to previous findings that have demonstrated that increasing age decreases the likelihood of forming romantic relationships (Skew et al. 2009). The current sample, however, was relatively young (mean age 23.2 years), which may explain why we did not find a reverse relationship between age and romantic relationship formation. With such a young sample, one might speculate that relatively older males may be more motivated, and hence more likely, to find a partner, compared to younger males, due to societal norms expecting them to "settle down" towards the end of young adulthood (Billari and Liefbroer 2010; Shulman and Connolly 2013). In addition, it takes some years following puberty to develop emotionally, intellectually 
Table 2 Characteristics associated with transitioning from single to a romantic relationship, $n=2146$. Reference category: single at T1 and T2, OR $=1$

\begin{tabular}{|c|c|c|c|}
\hline $\begin{array}{l}\text { Independent variables } \\
\text { (assessed at } \mathrm{T} 1 \text { ) }\end{array}$ & $\begin{array}{l}\text { Women }(n=1332) \\
\text { Single T1, romantic } \\
\text { relationship T2 } \\
\text { OR }(95 \% \mathrm{CI})\end{array}$ & $\begin{array}{l}\text { Men }(n=814) \\
\text { Single T1, romantic } \\
\text { relationship T2 } \\
\text { OR }(95 \% \text { CI })\end{array}$ & $\begin{array}{l}\text { Interaction effects of sex } \\
\text { Significance tests }\end{array}$ \\
\hline \multicolumn{4}{|l|}{ Demographics } \\
\hline AgeZ (age not $z$-scored) & $\begin{array}{l}0.87(0.73-1.04) \\
(0.97(0.93-1.01))\end{array}$ & $\begin{array}{l}1.25(1.03-1.51)^{*} \\
(1.05(1.01-1.10))\end{array}$ & $* *$ \\
\hline \multicolumn{4}{|l|}{ Country of birth } \\
\hline Outside Norway & 1.00 & 1.00 & \\
\hline Born in Norway & $1.57(0.80-3.10)$ & $0.97(0.47-1.97)$ & N.S. \\
\hline \multicolumn{4}{|l|}{ Parental status } \\
\hline No child/children & 1.00 & 1.00 & \\
\hline Have child/children & $3.42(1.42-8.22)^{* *}$ & $1.01(0.24-4.29)$ & N.S. \\
\hline \multicolumn{4}{|l|}{ Personality } \\
\hline ExtroversionZ & $1.20(1.02-1.40)^{*}$ & $1.58(1.26-1.99)^{* * *}$ & N.S. \\
\hline AgreeablenessZ & $1.06(0.91-1.23)$ & $0.94(0.76-1.15)$ & N.S. \\
\hline ConscientiousnessZ & $0.99(0.86-1.14)$ & $1.09(0.90-1.31)$ & N.S. \\
\hline NeuroticismZ & $1.23(1.06-1.42)^{* *}$ & $0.91(0.75-1.10)$ & $*$ \\
\hline OpennessZ & $1.02(0.89-1.18)$ & $1.37(1.12-1.68)^{* *}$ & $*$ \\
\hline \multicolumn{4}{|l|}{ Substance use } \\
\hline \multicolumn{4}{|l|}{ Nicotine use } \\
\hline Non-daily nicotine use & 1.00 & 1.00 & \\
\hline Daily nicotine use & $0.91(0.64-1.32)$ & $1.11(0.73-1.70)$ & N.S. \\
\hline \multicolumn{4}{|l|}{ Alcohol use } \\
\hline No alcohol use & 1.00 & 1.00 & \\
\hline Low-risk alcohol use $^{\mathrm{a}}$ & $2.57(1.08-6.12)^{*}$ & $1.46(0.47-4.52)$ & N.S. \\
\hline Hazardous alcohol use ${ }^{\mathrm{b}}$ & $2.82(1.18-6.71)^{*}$ & $2.86(0.96-8.50)$ & N.S. \\
\hline Harmful/dependent alcohol use ${ }^{c}$ & $3.01(1.12-8.09)^{*}$ & $2.10(0.64-6.86)$ & N.S. \\
\hline \multicolumn{4}{|l|}{ Illegal substance use last 6 months } \\
\hline No illegal substance use & 1.00 & 1.00 & \\
\hline Illegal substance use & $1.62(1.08-2.42)^{*}$ & $1.11(0.73-1.69)$ & N.S. \\
\hline Models, $p<0.001, \mathrm{df}=13$ for all & $\begin{array}{l}\chi^{2}=42.078 \\
\text { Cox and Snell }=0.031 ; \\
\quad \text { Nagelkerke } R^{2}=0.049\end{array}$ & $\begin{array}{l}\chi^{2}=61.077 \\
\text { Cox and Snell }=0.072 ; \\
\quad \text { Nagelkerke } R^{2}=0.115\end{array}$ & \\
\hline
\end{tabular}

$T 1$ time of the first survey, $T 2$ time of the second survey, OR odds ratio, $C I$ confidence interval, $Z z$-score, $* p<0.05, * * p<0.01, * * * p<0.001, N$.S. not significant. ${ }^{\mathrm{a}} 0<$ AUDIT $<8,{ }^{\mathrm{b}} 7<$ AUDIT $<16,{ }^{\mathrm{c}}$ AUDIT $>15$

and physically prior to parenthood (Bloom 2004), especially for men; hence, maturity may also explain why age was positively associated with romantic relationship formation in the male sample. Further, the older men in the current young sample may be closer to the age of peak fertility in men (i.e. 30-35 years) and thus be perceived as more attractive by women compared to the younger participants (Levitas et al. 2007). Moreover, the finding that increasing age predicted romantic relationship formation only among male participants is in line with the leading evolutionary-based hypothesis of increasing age as unlikely to hamper men's likelihood of romantic relationship formation to the same degree as women's due to sex differences in relation to age and fertility and the emphasis placed on a potential partner's resources (Buss 1989).

The finding that having children increased single, female, students' odds of forming a romantic relationship is another novel finding. The association between having children and forming a relationship had a moderate effect size, which indicates that having children is a rather important predictor of relationship formation, at least in the current female sample. Based on common evolutionary formulations suggesting men to highly value signs of fertility in a potential mate, one may further speculate that having children from a previous relationship could be perceived as a sign of fertility which may make women with children attractive to potential mates, but this 
tendency is not supported by previous findings. Previous studies have found single parents, and especially women, to be rated as less attractive and to be less likely to repartner, compared to singles without children (Bernhardt and Goldscheider 2002; Plumm et al. 2016; Skew et al. 2009; Sommer et al. 2013). One possible explanation for the discrepancy between the current results and previous ones may be that previous studies mostly focus on attractiveness ratings given to single parents (which is one but not the only factor affecting the likelihood of repartnering). Furthermore, the one study investigating the likelihood of repartnering had an older sample than the current sample and is from 2002 (Bernhardt and Goldscheider 2002). Having children may not be perceived as a sign of fertility among older individuals and perceptions of single parents may have become more liberal since 2002; hence, sample characteristics and the time of the current study may explain discrepancies between the findings of Bernhardt and Goldscheider (2002) and the current findings. In addition to children as a signal of fertility, other explanations for why the single mothers in our sample had an increased likelihood of entering relationships include the supposition that they may have a greater need for support and thus may be more motivated to find a partner compared to single and childless women. In addition, it should be noted that few women in the current sample had children. Those who have children may seek other characteristics in their partner and meet other potential partners, compared to those without children (e.g. be more positive to other single parents) (Plumm et al. 2016). Given that few women had children, the ones who did may have less competition regarding their preferred mates, and hence more success in obtaining a partner.

\section{Extroversion, Neuroticism and Openness}

Higher extroversion and openness scores (the latter among men only) predicted romantic relationship formation, which are both novel findings. The finding of extroversion as a predictor of relationship formation, however, is in line with one study finding sociability (i.e. a sub-facet of extroversion) to be a predictor of relationship formation (Neyer and Lehnart 2007). Individuals with higher extroversion and openness scores have previously been shown to be rated as more attractive as romantic partners (Botwin et al. 1997; Figueredo et al. 2006). The increased attractiveness ratings given to potential mates with higher extroversion and openness scores may in part explain why student participants with these characteristics were more likely to form romantic relationships. Further, extroverted individuals tend to have an easier time forming new social relationships in general and have better social skills, compared to less extroverted individuals (Anderson et al. 2001; Argyle and Lu 1990). Larger social networks and good social skills are likely to facilitate meeting and attracting potential mates. Individuals with high openness scores are characterised by a high tolerance for and interest in novel experiences (McCrae and Costa 1997; McCrae and John 1992). The participants with high openness scores' increased likelihood of entering into romantic relationships may be explained by the fact that their openness to new experiences leads to more frequent exposure to new people and circumstances, a quintessential condition for meeting potential romantic partners.

Higher neuroticism scores among women were related to an increased likelihood of forming romantic relationships, which is in line with a previous study that also found neuroticism to predict relationship formation (Neyer and Lehnart 2007). The finding that neuroticism may increase the likelihood of relationship formation only for women, however, is a novel finding. The observed association between higher neuroticism scores and relationship formation run counter to studies finding neuroticism to be a less desirable trait in potential partners (Botwin et al. 1997; Figueredo et al. 2006). Neuroticism is associated with poorer social support and higher levels of negative affect and anxiety (McCrae and John 1992; Russell et al. 1997). Accordingly, neurotic individuals may be more motivated to find a romantic partner in order to obtain emotional support, which may explain why the women in our sample with higher neuroticism scores were more likely to enter into a romantic relationship. Furthermore, higher neuroticism scores are associated with increased emotional expressiveness, which may aid the formation of social bonds in general (including romantic ones) (Judge et al. 2009).

\section{Alcohol Use and Illegal Drug Use}

The female students in our sample who reported a low-risk, hazardous or harmful/dependent alcohol use had a higher likelihood of romantic relationship formation, compared to the female students who did not drink. The association between harmful/dependent alcohol use and relationship formation had a moderate effect size, which indicates that quite heavy alcohol use is a considerable predictor of relationship formation among single, female students. Further, the single female students who had used illegal substances during the previous 6 months at T1 had a higher likelihood of being in a romantic relationship at T2, compared to the female students who had not used illegal substances the last 6 months at T1. Very few studies have investigated the relationship between substance use and forming romantic relationships. The current results do, however, contrast with the trend found in previous research suggesting that alcohol and illegal substance use reduce the likelihood of romantic relationship formation (Leonard and Rothbard 1999). The design of the current study is not conducive to drawing conclusions regarding the mechanisms behind the relationship between substance use and relationship formation. For alcohol use, one possible explanation may 
be related to alcohol use and in part heavy alcohol use, being the norm among Norwegian students (Erevik et al. 2017a; Nedregård and Olsen 2014). The finding that female students with high alcohol use have an increased likelihood of forming a romantic relationship may thus be explained by them being more in contact with potential partners at parties, etc., in contrast to the female students who do not drink alcohol at all. Further, excessive use of illegal drugs appears to be uncommon in the Norwegian student population (Erevik et al. 2017b; Nedregård and Olsen 2014); thus the students in our sample who report using illegal substances probably did not have a substance use disorder per se, and the results may have been different if they had. Some use of illegal substances indicate a willingness to take risks, a trait which is further considered as attractive in, at least short-term, romantic partners (Bassett and Moss 2004). The perceived attractiveness of individuals who are willing to take risks may further explain why female students using illegal substances were more likely to form romantic relationships.

Moreover, some of the effects of psychoactive substances may also explain why alcohol and illegal substance use were predictors of later relationship formation in women. Some psychoactive substances (including alcohol) are known to aid flirtation because they can make the users less inhibited, boost self-confidence and elevate positive affect (e.g. infatuation) (Abrahamson 2004; Ter Bogt and Engels 2005). This may help to explain why alcohol and illegal substance use predicted romantic relationship formation among women. Finally, it is possible that the women who used more alcohol or illegal substances had a greater need for support compared to those having a lower degree of substance use as substance use generally predicts emotional problems (Anthony et al. 1989; Grant et al. 2004; Lev-Ran et al. 2014). An increased need for companionship and support may further make these women more motivated to find a partner and perhaps lower their expectations for a partner and thereby explain their increased likelihood of romantic relationship formation.

\section{Sex Differences}

Beyond extroversion predicting romantic relationship formation among both women and men, the other individual characteristics predicting romantic relationship formation were different for the two sexes. The characteristics that were exclusively associated with forming romantic relationships among female students were having children, higher scores on neuroticism, higher alcohol use and having used illegal substances during the previous 6 months at T1. These characteristics could all be related to a greater need for companionship and support, and women with these characteristics may be more motivated to find a partner in order to find such companionship and support. Women signalling such needs may be more accepted by potential male partners as opposed to men seeking such resources, due to evolutionary history and prevailing gender roles, where women historically are theorised to have needed more protection from dangers than men, and men, conversely, appreciated and accepted taking on protective roles (Buss 1998; Cross and Overall 2018). In addition, the findings for female students are further in line with theories of female choice and parental investment (Perper 1989; Trivers 1972). These theories suggest that women with less attractive characteristics will have an easier time finding a partner compared to men with less attractive characteristics due to differences in the minimum level of investment in potential offspring, which makes women (i.e. the most investing sex) more exacting in choosing a mate.

Among the single male students, on the other hand, increasing age and higher scores on openness predicted relationship formation. These characteristics can be regarded as indicators of resource acquisition potential, because relatively older men are likely to be in a better financial position or are expected to acquire such a position sooner in contrast to younger men. Likewise, openness is associated with intelligence which is further associated with achieving higher salaries (Ashton et al. 2000; Buss 1989; Strenze 2007). The explanation for why these two characteristics were not associated with being in a relationship 1 year later, among women, may be related to gender roles and evolutionary factors as well, since men (and not women) have historically been expected to provide resources to support the upbringing of children (Bertrand et al. 2015; Buss 1989, 1998).

The sex difference regarding the association between substance use and forming a romantic relationship may also derive from a sex imbalance in milieus where substance use is the norm. Men have consistently been found to have a higher substance use compared to women (Erevik et al. 2017a, 2017b). Single female students with high substance use's increased likelihood of forming a romantic relationship might thus be explained by them being in a setting with more potential mates and fewer female competitors, while men, on the other hand, may meet fewer potential mates and have more male competitors in settings where substances are used.

\section{Limitations and Strengths}

The current study has some limitations that should be noted. For one, the design of the study precludes conclusions regarding the mechanisms behind the relationship formations (e.g. whether a characteristic predicts relationship formation because individuals with this characteristic are more desirable or because they have lower expectations for a partner). Underlying mechanisms need to be elucidated by future research. Further, certain potentially important differences within the sample were not measured or controlled for (i.e. in terms of sexual orientation, gender identity, reasons for singledom and seriousness of the newly formed romantic commitment). 
The participants were not asked about sexual orientation or gender identity. This is an important limitation as forming a romantic relationship might be predicted by other individual characteristics among LBGT individuals compared to heterosexual and/or cisgender individuals (Gobrogge et al. 2007). In addition, the participants' reasons for singlehood were not assessed, and it is possible that different characteristics may have predicted relationship formation among those who perceived themselves as voluntarily single versus those who did not (Adamczyk 2017). Another limitation is related to the fact that we do not know the duration of the romantic relationships the students entered, and formation of short-term relationships may be predicted by other characteristics than the characteristics predicting long-term relationship formation. The participants who had entered into romantic relationships, however, are likely to have had the intentions to enter into long-term relationships, because Norwegians normally understand the phrase "being in a relationship" as referring to a serious, monogamous, interpersonal, long-term commitment.

Furthermore, the included variables far from explained all variance in the romantic relationship formation outcome. Hence, it is important that future studies explore other possible predictors, where in particular, including variables such as physical attractiveness, expected and actual income and characteritics of family of origin might increase explanatory power (Buss 1989; Draper and Harpending 1982). In addition, having a shorter timeframe between the measurement of predictors and the measurement of outcome than the current study's timeframe of 1 year may also increase explanatory power because both personality and substance use may change somewhat during the period of 1 year due to the formative nature of young adulthood/higher education (Neyer and Lehnart 2007; Schulenberg and Maggs 2002). It should also be noted that there were fewer men than women in the current study, and some associations may have gone undetected in the male group due to the lower statistical power in this group.

Finally, the generalisability of the current study may be perceived as a potential limitation. Nevertheless, we believe that the current results are likely to be applicable to the Norwegian student population because the response rate at T1 (i.e. $39.4 \%$ ) is relatively high compared to similar studies, and because the participants had similar sex, age, relationship status and alcohol use characteristics as those reported in other studies on Norwegian students (Nedregård and Olsen 2014; Sheehan 2001; Statistisk sentralbyrå [Statistics Norway] 2017). The current results, however, may not be transferable to other populations. Given that the sample consisted solely of college/university students and since college/university students are known to have certain demographic and personality traits that may make them more attractive as romantic partners (Botwin et al. 1997; Shiner et al. 2003), the results may be influenced by the phenomena of conditioning on a collider where associations between variables in reality are explained by characteristics of the specific sample (Rohrer 2017). Consequentially, future studies should investigate whether the current findings hold true in other populations as well. Even though the current results are not directly generalisable to populations other than the Norwegian student population, the results are likely to be relevant for other populations since some trends in romantic relationship formation are theorised to be universal (Buss 1989).

The present study has some noteworthy strengths such as the large sample size and the number of variables included and controlled for. The large sample size minimises the risk of type II errors, and the high number of variables included makes it less likely that the reported associations are explained by alternative third variables. However, the high number of significance tests conducted also entails an increased risk of committing type I errors. The longitudinal design is another important asset of the current study, as this design supports interpretations regarding directionality. Finally, the current study assessed associations that previously have not been investigated and contributes as such several novel findings regarding an important human topic.

\section{Conclusions}

The associations between demographics, personality and substance-use characteristics on the one hand, and romantic relationship formation on the other, has only to a limited extent been investigated in previous studies and the current study contributes as such with several novel findings. Factors from all three groups of predictors turned out to be significant predictors, i.e. age, having children, extroversion, neuroticism, openness, alcohol use and illegal substance use. With one exception, there were sex differences in these relationships. A general pattern appeared to be that women with characteristics related to a need for support (e.g. neuroticism) were more likely to enter a romantic relationship, whereas characteristics associated with increased resource acquisition potential (e.g. openness) predicted romantic relationship formation in men. The general pattern of findings is in line with established evolutionary theories such as the sexual strategies theory and the parental investment theory. Future studies should investigate whether the current findings hold true in other populations.

Acknowledgements We want to thank Trude Remme for her help in setting up the online surveys. We also want to thank two anonymous reviewers for the helpful comments and suggestions that improved this paper.

Funding This study was funded by a $\mathrm{PhD}$ grant given to the first author from the University of Bergen, Norway and Bergen municipality. No 
specific grant from any funding agency in the public, commercial, or notfor-profit sectors was received.

\section{Compliance with Ethical Standards}

Conflict of Interest The authors declare that they have no conflict of interest.

Ethical Approval All procedures performed in studies involving human participants were in accordance with the ethical standards of the institutional and/or national research committee and with the 1964 Helsinki Declaration and its later amendments or comparable ethical standards.

Open Access This article is distributed under the terms of the Creative Commons Attribution 4.0 International License (http:// creativecommons.org/licenses/by/4.0/), which permits unrestricted use, distribution, and reproduction in any medium, provided you give appropriate credit to the original author(s) and the source, provide a link to the Creative Commons license, and indicate if changes were made.

\section{References}

Abrahamson, M. (2004). Alcohol in courtship contexts: focus-group interviews with young Swedish women and men. Contemporary Drug Problems, 31(1), 3-29. https://doi.org/10.1177/ 009145090403100102.

Adamczyk, K. (2017). Voluntary and involuntary singlehood and young adults' mental health: an investigation of mediating role of romantic loneliness. Current Psychology, 36(4), 888-904. https://doi.org/10. 1007/s12144-016-9478-3.

Allison, P. D. (2001). Missing data. CA, US: Sage.

Allison, P. D. (2014). Listwise deletion is not evil. Retrieved from http:// statisticalhorizons.com/listwise-deletion-its-not-evil

Anderson, C., John, O. P., Keltner, D., \& Kring, A. M. (2001). Who attains social status? Effects of personality and physical attractiveness in social groups. Journal of Personality and Social Psychology, 81(1), 116-132. https://doi.org/10.1037/0022-3514.81.1.116.

Anthony, J. C., Tien, A. Y., \& Petronis, K. R. (1989). Epidemiologic evidence on cocaine use and panic attacks. American Journal of Epidemiology, 129(3), 543-549. https://doi.org/10.1093/ oxfordjournals.aje.a115166.

Argyle, M., \& Lu, L. (1990). Happiness and social skills. Personality and Individual Differences, 11(12), 1255-1261. https://doi.org/10.1016/ 0191-8869(90)90152-H.

Ashton, M. C., Lee, K., Vernon, P. A., \& Jang, K. L. (2000). Fluid intelligence, crystallized intelligence, and the openness/intellect factor. Journal of Research in Personality, 34(2), 198-207. https://doi. org/10.1006/jrpe.1999.2276.

Averett, S. L., Sikora, A., \& Argys, L. M. (2008). For better or worse: relationship status and body mass index. Economics \& Human Biology, 6(3), 330-349. https://doi.org/10.1016/j.ehb.2008.07.003.

Babor, T. F., Higgins-Biddle, J. C., Saunders, J. B., \& Monteiro, M. G. (2001). The alcohol use disorders identification test: guidelines for use in primary care. www.who.int: World Health Organization.

Bachman, J. G., Wadsworth, K. N., O’Malley, P. M., \& Johnston, L. D. (1997). Smoking, drinking, and drug use in young adulthood: the impacts of new freedoms and new responsibilities. Hillsdale, $\mathrm{NJ}$ : Lawrence Erlbaum Assocs., Inc..

Bassett, J. F., \& Moss, B. (2004). Men and women prefer risk takers as romantic and nonromantic partners. Current Research in Social Psychology, 9(10), 135-144.
Bernhardt, E., \& Goldscheider, F. (2002). Children and union formation in Sweden. European Sociological Review, 18(3), 289-299. https:// doi.org/10.1093/esr/18.3.289.

Bertrand, M., Kamenica, E., \& Pan, J. (2015). Gender identity and relative income within households. The Quarterly Journal of Economics, 130(2), 571-614. https://doi.org/10.1093/qje/qjv001.

Billari, F. C., \& Liefbroer, A. C. (2010). Towards a new pattern of transition to adulthood? Advances in Life Course Research, 15(2-3), 59-75. https://doi.org/10.1016/j.alcr.2010.10.003.

Bloom, D. F. (2004). Is acne really a disease?: a theory of acne as an evolutionarily significant, high-order psychoneuroimmune interaction timed to cortical development with a crucial role in mate choice. Medical Hypotheses, 62(3), 462-469. https://doi.org/10.1016/j. mehy.2003.11.003.

Bohn, M. J., Babor, T. F., \& Kranzler, H. R. (1995). The alcohol use disorders identification test (AUDIT): validation of a screening instrument for use in medical settings. Journal of Studies on Alcohol, 56(4), 423-432. https://doi.org/10.15288/jsa.1995.56.423.

Botwin, M. D., Buss, D. M., \& Shackelford, T. K. (1997). Personality and mate preferences: five factors in mate selection and marital satisfaction. Journal of Personality, 65(1), 107-136. https://doi.org/10. 1111/j.1467-6494.1997.tb00531.x.

Buss, D. M. (1989). Sex differences in human mate preferences: evolutionary hypotheses tested in 37 cultures. Behavioral and Brain Sciences, 12(1), 1-14. https://doi.org/10.1017/ S0140525X00023992.

Buss, D. M. (1998). Sexual strategies theory: historical origins and current status. Journal of Sex Research, 35(1), 19-31. https://doi.org/ $10.1080 / 00224499809551914$.

Buss, D. M. (2007). The evolution of human mating strategies. In S. W. Gangestad \& J. A. Simpson (Eds.), The evolution of mind: fundamental questions and controversies (pp. 375-382). New York, NY: Guilford Press.

Buss, D. M. (2009). How can evolutionary psychology successfully explain personality and individual differences? Perspectives on Psychological Science, 4(4), 359-366. https://doi.org/10.1111/j. 1745-6924.2009.01138.x.

Buss, D. M., \& Schmitt, D. P. (1993). Sexual strategies theory: an evolutionary perspective on human mating. Psychological Review, 100(2), 204-232. https://doi.org/10.1037/0033-295X.100.2.204.

Chen, H., Cohen, P., \& Chen, S. (2010). How big is a big odds ratio? Interpreting the magnitudes of odds ratios in epidemiological studies. Communications in Statistics-Simulation and Computation, 39(4), 860-864. https://doi.org/10.1080/03610911003650383.

Cross, E. J., \& Overall, N. C. (2018). Women's attraction to benevolent sexism: needing relationship security predicts greater attraction to men who endorse benevolent sexism. European Journal of Social Psychology, 48(3), 336-347. https://doi.org/10.1002/ejsp.2334.

Desmond, M. (1967). The naked ape: a zoologist's study of the human animal. London, UK: Cape.

Dixon, R. B. (1971). Explaining cross-cultural variations in age at marriage and proportions never marrying. Population Studies, 25(2), 215-233. https://doi.org/10.2307/2173211.

Donnellan, M. B., Oswald, F. L., Baird, B. M., \& Lucas, R. E. (2006). The mini-IPIP scales: tiny-yet-effective measures of the big five factors of personality. Psychological Assessment, 18(2), 192-203. https://doi.org/10.1037/1040-3590.18.2.192.

Draper, P., \& Harpending, H. (1982). Father absence and reproductive strategy: an evolutionary perspective. Journal of Anthropological Research, 38(3), 255-273. https://doi.org/10.1086/jar.38.3. 3629848.

Dudley, R. (2002). Fermenting fruit and the historical ecology of ethanol ingestion: is alcoholism in modern humans an evolutionary hangover? Addiction, 97(4), 381-388. https://doi.org/10.1046/j.13600443.2002.00002.x. 
Dush, C. M. K., \& Amato, P. R. (2005). Consequences of relationship status and quality for subjective well-being. Journal of Social and Personal Relationships, 22(5), 607-627. https://doi.org/10.1177/ 0265407505056438.

Erevik, E. K., Pallesen, S., Vedaa, Ø., Andreassen, C. S., \& Torsheim, T. (2017a). Alcohol use among Norwegian students: demographics, personality and psychological health correlates of drinking patterns. Nordic Studies on Alcohol and Drugs, 34(5), 415-429. https://doi. org/10.1177/1455072517709918.

Erevik, E. K., Torsheim, T., Andreassen, C. S., Vedaa, Ø., \& Pallesen, S. (2017b). Recurrent cannabis use among Norwegian students: prevalence, characteristics, and polysubstance use. Nordic Studies on Alcohol and Drugs, 34(6), 497-510. https://doi.org/10.1177/ 1455072517743427.

Farthing, G. W. (2005). Attitudes toward heroic and nonheroic physical risk takers as mates and as friends. Evolution and Human Behavior, 26(2), 171-185. https://doi.org/10.1016/j.evolhumbehav.2004.08. 004 .

Ferguson, C. J. (2009). An effect size primer: a guide for clinicians and researchers. Professional Psychology: Research and Practice, 40(5), 532-538. https://doi.org/10.1037/a0015808.

Figueredo, A. J., Sefcek, J. A., \& Jones, D. N. (2006). The ideal romantic partner personality. Personality and Individual Differences, 41(3), 431-441. https://doi.org/10.1016/j.paid.2006.02.004.

Frank, O., Bianchi, P. G., \& Campana, A. (1994). The end of fertility: age, fecundity and fecundability in women. Journal of Biosocial Science, 26(3), 349-368. https://doi.org/10.1017/S002193200002143X.

$\mathrm{Fu}, \mathrm{H} .$, \& Goldman, N. (1996). Incorporating health into models of marriage choice: demographic and sociological perspectives. Journal of Marriage and the Family, 58(3), 740-758. https://doi.org/10.2307/ 353733.

Gobrogge, K. L., Perkins, P. S., Baker, J. H., Balcer, K. D., Breedlove, S. M., \& Klump, K. L. (2007). Homosexual mating preferences from an evolutionary perspective: sexual selection theory revisited. Archives of Sexual Behavior, 36(5), 717-723. https://doi.org/10. 1007/s10508-007-9216-x.

Grant, B. F., Stinson, F. S., Dawson, D. A., Chou, S. P., Dufour, M. C., Compton, W., Pickering, R. P., \& Kaplan, K. (2004). Prevalence and co-occurrence of substance use disorders and independent mood and anxiety disorders - results from the national epidemiologic survey on alcohol and related conditions. Archives of General Psychiatry, 61(8), 807-816. https://doi.org/10.1001/archpsyc.61.8.807.

Hamilton, W. D. (1964). The genetical evolution of social behaviour II. Journal of Theoretical Biology, 7(1), 17-52. https://doi.org/10.1016/ 0022-5193(64)90039-6.

Henderson, V. R., Hennessy, M., Barrett, D. W., Curtis, B., McCoy-Roth, M., Trentacoste, N., \& Fishbein, M. (2005). When risky is attractive: sensation seeking and romantic partner selection. Personality and Individual Differences, 38(2), 311-325. https://doi.org/10.1016/j. paid.2004.04.010.

Judge, T. A., Piccolo, R. F., \& Kosalka, T. (2009). The bright and dark sides of leader traits: a review and theoretical extension of the leader trait paradigm. The Leadership Quarterly, 20(6), 855-875. https:// doi.org/10.1016/j.leaqua.2009.09.004.

Kandel, D. B., Davies, M., Karus, D., \& Yamaguchi, K. (1986). The consequences in young adulthood of adolescent drug involvement: an overview. Archives of General Psychiatry, 43(8), 746-754. https://doi.org/10.1001/archpsyc.1986.01800080032005.

Kidd, S. A., Eskenazi, B., \& Wyrobek, A. J. (2001). Effects of male age on semen quality and fertility: a review of the literature. Fertility and Sterility, 75(2), 237-248. https://doi.org/10.1016/S0015-0282(00) 01679-4.

Kiecolt-Glaser, J. K., \& Newton, T. L. (2001). Marriage and health: his and hers. Psychological Bulletin, 127(4), 472-503. https://doi.org/ 10.1037/0033-2909.127.4.472.
Kirkpatrick, R.C., Blackwood, E., Dickemann, J. M., Jones, D., Muscarella, F., Vasey, P. L., et al. (2000). The evolution of human homosexual behavior. Current Anthropology, 41(3), 385-413. https://doi.org/10.1086/300145.

Larsen, R. J., Buss, D. M., \& Wismeijer, A. (2013). Personality psychology: Domains of knowledge about human nature. Berkshire, UK: McGraw Hill Education.

Leonard, K. E., \& Rothbard, J. C. (1999). Alcohol and the marriage effect. Journal of Studies on Alcohol, Supplement(13), 139-146. https://doi.org/10.15288/jsas.1999.s13.139.

Lev-Ran, S., Roerecke, M., Le Foll, B., George, T., McKenzie, K., \& Rehm, J. (2014). The association between cannabis use and depression: a systematic review and meta-analysis of longitudinal studies. Psychological Medicine, 44(4), 797-810. https://doi.org/10.1017/ S0033291713001438.

Levitas, E., Lunenfeld, E., Weisz, N., Friger, M., \& Potashnik, G. (2007). Relationship between age and semen parameters in men with normal sperm concentration: analysis of 6022 semen samples. Andrologia, 39(2), 45-50. https://doi.org/10.1111/j.1439-0272.2007.00761.x.

Long, J. S. (1997). Regression models for categorical and limited dependent variables. Thousand Oaks, CA: Sage Publications.

Lovejoy, C. O. (1981). The origin of man. Science, 211(4480), 341-350. https://doi.org/10.1126/science.211.4480.341.

Lukas, D., \& Clutton-Brock, T. H. (2013). The evolution of social monogamy in mammals. Science, 341(6145), 526-530. https://doi.org/ 10.1126/science. 1238677 .

Manning, W. D., Brown, S. L., \& Payne, K. K. (2014). Two decades of stability and change in age at first union formation. Journal of Marriage and Family, 76(2), 247-260. https://doi.org/10.1111/ jomf. 12090.

McCrae, R. R. (2002). Cross-cultural research on the five-factor model of personality. Online Readings in Psychology and Culture, 4(4), 1-12. https://doi.org/10.9707/2307-0919.1038.

McCrae, R. R., \& Costa, P. T. (1997). Conceptions and correlates of openness to experience. In R. Hogan, J. Johnson, \& S. Briggs (Eds.), Handbook of personality psychology (pp. 825-847). San Diego, CA: Elsevier.

McCrae, R. R., Costa, P. T., de Lima, M. P., Simões, A., Ostendorf, F., Angleitner, A., . . . Barbaranelli, C. (1999). Age differences in personality across the adult life span: parallels in five cultures. Developmental Psychology, 35(2), 466-477. https://doi.org/10. 1037/0012-1649.35.2.466

McCrae, R. R., \& John, O. P. (1992). An introduction to the 5-factor model and its applications. Journal of Personality, 60(2), 175215. https://doi.org/10.1111/j.1467-6494.1992.tb00970.x.

Nargund, G. (2009). Declining birth rate in developed countries: a radical policy re-think is required. Facts, Views \& Vision in ObGyn, 1(3), 191-193.

Nedregård, T., \& Olsen, R. (2014). Studentenes helse- og trivselsundersøkelse 2014. [Students' Health and Wellbeing Survey 2014]. Retrieved from: http://www.google.no/url?sa= $\mathrm{t} \& \mathrm{rct}=\mathrm{j} \& \mathrm{q}=\& \mathrm{esrc}=\mathrm{s} \& \mathrm{frm}=1 \&$ source $=\mathrm{web} \& \mathrm{~cd}=1 \& \mathrm{ved}=$ 0CB0QFjAA\&url=http $\% 3 \mathrm{~A} \% 2 \mathrm{~F} \% 2 \mathrm{Fwww} . v t b e r g e n . n o \% 2 \mathrm{Fwp}-$ content\%2Fuploads\%2F2013\%2F10\%2FVT0614_6214 SHoT2014.pdf\&ei=w21HVZvGEIOvswG8woGQDw\&usg= AFQjCNESqBK5IcWkVZUNzMxPA7ANwM2HzA\&sig2= CahhInSPe7w4ZBNAUWdXiA.

Nettle, D. (2006). The evolution of personality variation in humans and other animals. American Psychologist, 61(6), 622-631. https://doi. org/10.1037/0003-066X.61.6.622.

Newcomb, M. D., \& Bentler, P. M. (1987). Changes in drug use from high school to young adulthood: effects of living arrangement and current life pursuit. Journal of Applied Developmental Psychology, 8(3), 221-246. https://doi.org/10.1016/0193-3973(87)90001-3.

Neyer, F. J., \& Asendorpf, J. B. (2001). Personality-relationship transaction in young adulthood. Journal of Personality and Social 
Psychology, 81(6), 1190-1204. https://doi.org/10.1037/0022-3514. 81.6.1190.

Neyer, F. J., \& Lehnart, J. (2007). Relationships matter in personality development: evidence from an 8-year longitudinal study across young adulthood. Journal of Personality, 75(3), 535-568. https:// doi.org/10.1111/j.1467-6494.2007.00448.x.

Opie, C., Atkinson, Q. D., Dunbar, R. I., \& Shultz, S. (2013). Male infanticide leads to social monogamy in primates. Proceedings of the National Academy of Sciences, 110(33), 13328-13332. https:// doi.org/10.1073/pnas.1307903110.

Pedersen, W., Tutenges, S., \& Sandberg, S. (2017). The pleasures of drunken one-night stands: assemblage theory and narrative environments. International Journal of Drug Policy, 49, 160-167. https:// doi.org/10.1016/j.drugpo.2017.08.005.

Perper, T. (1989). Theories and observations on sexual selection and female choice in human beings. Medical Anthropology, 11(4), 409-454. https://doi.org/10.1080/01459740.1989.9966006.

Petraitis, J. M., Lampman, C. B., Boeckmann, R. J., \& Falconer, E. M. (2014). Sex differences in the attractiveness of hunter-gatherer and modern risks. Journal of Applied Social Psychology, 44(6), 442 453. https://doi.org/10.1111/jasp.12237.

Plumm, K. M., Sommer, S., Uhl, C., \& Stone, K. (2016). Single parent seeking single parent? The effects of parental and previous relationship status on perceptions of online dating profiles. Journal of Relationships Research, 7, 1-12. https://doi.org/10.1017/jrr.2016. 10.

Qian, Z., Glick, J. E., \& Batson, C. D. (2012). Crossing boundaries: nativity, ethnicity, and mate selection. Demography, 49(2), 651675. https://doi.org/10.1007/s13524-012-0090-3.

Rehm, J., Room, R., Graham, K., Monteiro, M., Gmel, G., \& Sempos, C. T. (2003). The relationship of average volume of alcohol consumption and patterns of drinking to burden of disease: an overview. Addiction, 98(9), 1209-1228. https://doi.org/10.1046/j.1360-0443. 2003.00467.x.

Rehm, J., Taylor, B., \& Room, R. (2006). Global burden of disease from alcohol, illicit drugs and tobacco. Drug and Alcohol Review, 25(6), 503-513. https://doi.org/10.1080/09595230600944453.

Roberts, B. W., Kuncel, N. R., Shiner, R., Caspi, A., \& Goldberg, L. R. (2007). The power of personality: the comparative validity of personality traits, socioeconomic status, and cognitive ability for predicting important life outcomes. Perspectives on Psychological Science, 2(4), 313-345. https://doi.org/10.1111/j.1745-6916.2007.00047.x.

Rohrer, J. (2017). That one weird third variable problem nobody ever mentions: conditioning on a collider. Retrieved from http://www. the100.ci/2017/03/14/that-one-weird-third-variable-problemnobody-ever-mentions-conditioning-on-a-collider/

Russell, D. W., Booth, B., Reed, D., \& Laughlin, P. R. (1997). Personality, social networks, and perceived social support among alcoholics: a structural equation analysis. Journal of Personality, 65(3), 649-692. https://doi.org/10.1111/j.1467-6494.1997.tb00330.x.

Schulenberg, J. E., \& Maggs, J. L. (2002). A developmental perspective on alcohol use and heavy drinking during adolescence and the transition to young adulthood. Journal of Studies on Alcohol, Supplement(14), 54-70. https://doi.org/10.15288/jsas.2002.s14.54.

Shackelford, T. K., Schmitt, D. P., \& Buss, D. M. (2005). Universal dimensions of human mate preferences. Personality and Individual Differences, 39(2), 447-458. https://doi.org/10.1016/j. paid.2005.01.023.

Shaver, P. R., \& Brennan, K. A. (1992). Attachment styles and the "Big Five" personality traits: their connections with each other and with romantic relationship outcomes. Personality and Social Psychology Bulletin, 18(5), 536-545. https://doi.org/10.1177/ 0146167292185003.

Sheehan, K. B. (2001). E-mail survey response rates: a review. Journal of Computer-Mediated Communication, 6(2), 0-0. https://doi.org/10. 1111/j.1083-6101.2001.tb00117.x.
Shiner, R. L., Masten, A. S., \& Roberts, J. M. (2003). Childhood personality foreshadows adult personality and life outcomes two decades later. Journal of Personality, 71(6), 1145-1170. https://doi.org/10. 1111/1467-6494.7106010.

Shulman, S., \& Connolly, J. (2013). The challenge of romantic relationships in emerging adulthood: reconceptualization of the field. Emerging Adulthood, 1(1), 27-39. https://doi.org/10.1177/ 2167696812467330.

Skew, A., Evans, A., \& Gray, E. (2009). Repartnering in the United Kingdom and Australia. Journal of Comparative Family Studies, 40(4), 563-585.

Skirbekk, V., \& Blekesaune, M. (2014). Personality traits increasingly important for male fertility: evidence from Norway. European Journal of Personality, 28(6), 521-529. https://doi.org/10.1002/per.1936.

Soler, C., Nunez, M., Gutierrez, R., Nunez, J., Medina, P., Sancho, M., et al. (2003). Facial attractiveness in men provides clues to semen quality. Evolution and Human Behavior, 24(3), 199-207. https://doi. org/10.1016/S1090-5138(03)00013-8.

Sommer, S., Plumm, K. M., Terrance, C. A., \& Tubré, T. (2013). Perceptions of younger single adults as a function of their gender and number of children. The Journal of General Psychology, 140(2), 87-109. https://doi.org/10.1080/00221309.2013.769931.

Soons, J. P., \& Liefbroer, A. C. (2008). Together is better? Effects of relationship status and resources on young adults' well-being. Journal of Social and Personal Relationships, 25(4), 603-624. https://doi.org/10.1177/0265407508093789.

Statistisk sentralbyrå [Statistics Norway]. (2017). Studenter i høyere utdanning [Students in higher education]. Retrieved from https:// www.ssb.no/utdanning/statistikker/utuvh

Strenze, T. (2007). Intelligence and socioeconomic success: a metaanalytic review of longitudinal research. Intelligence, 35(5), 401426. https://doi.org/10.1016/j.intell.2006.09.004.

Sullivan, R. J., \& Hagen, E. H. (2002). Psychotropic substance-seeking: evolutionary pathology or adaptation? Addiction, 97(4), 389-400. https://doi.org/10.1046/j.1360-0443.2002.00024.x.

Taylor, J., Fulop, N., \& Green, J. (1999). Drink, illicit drugs and unsafe sex in women. Addiction, 94(8), 1209-1218. https://doi.org/10. 1046/j.1360-0443.1999.948120911.x.

Ter Bogt, T. F. M., \& Engels, R. C. M. E. (2005). "Partying” hard: party style, motives for and effects of MDMA use at rave parties. Substance Use \& Misuse, 40(9-10), 1479-1502. https://doi.org/10. 1081/JA-200066822.

Thiessen, D., \& Gregg, B. (1980). Human assortative mating and genetic equilibrium: an evolutionary perspective. Ethology and Sociobiology, 1(2), 111-140. https://doi.org/10.1016/01623095(80)90003-5.

Trivers, R. L. (1972). Parental investment and sexual selection. In B. Campbell (Ed.), Sexual selection and the descent of man (pp. 136179). Chicago, IL: Aldine.

Waal, F. B. (2006). Our inner ape: the best and worst of human nature. London: Granta Books.

Waal, F. B., \& Gavrilets, S. (2013). Monogamy with a purpose. Proceedings of the National Academy of Sciences, 110(38), 15167-15168. https://doi.org/10.1073/pnas.1315839110.

Wallace, W. H. B., \& Kelsey, T. W. (2010). Human ovarian reserve from conception to the menopause. PLoS One, 5(1), e8772. https://doi. org/10.1371/journal.pone.0008772.

Zahavi, A. (1975). Mate selection - a selection for a handicap. Journal of Theoretical Biology, 53(1), 205-214. https://doi.org/10.1016/00225193(75)90111-3.

Publisher's Note Springer Nature remains neutral with regard to jurisdictional claims in published maps and institutional affiliations. 